\title{
A comparison of laboratory, clinical, and self-report measures of prospective memory in healthy adults and individuals with brain injury
}

\author{
Sarah A. Raskin ${ }^{a}$, David H. K. Shum ${ }^{\mathrm{b}, \mathrm{c}}$, Judi Ellis ${ }^{\mathrm{d}}$, Antonina Pereira ${ }^{\mathrm{e}}$ and Ginger Mills ${ }^{\mathrm{f}}$

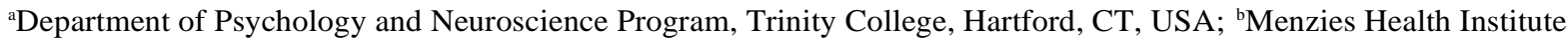 \\ Queensland and School of Applied Psychology, Griffith University, Brisbane, Gold Coast, QL, Australia; \\ ${ }^{\mathrm{c}}$ Neuropsychology and Applied Cognitive Neuroscience Laboratory, CAS Key Laboratory of Mental Health, Institute of \\ Psychology, Chinese Academy of Sciences, Beijing, China; d Department of Psychology, University of Reading, \\ Reading, UK; ${ }^{e}$ Department of Psychology and Counselling, University of Chichester, Chichester, UK; ${ }^{\mathrm{f}}$ Department of \\ Clinical Psychology, University of Hartford, Hartford, CT, USA
}

\begin{abstract}
Individuals with traumatic brain injury (TBI) have demonstrated deficits in prospective memory (PM) functioning when compared to healthy adults. These deficits have been measured using laboratory measures, clinical measures, and self-report questionnaires. However, PM has been shown to involve multiple cognitive processes and have a variety of stages. Thus, it is not known whether these measures all assess the same aspects of PM. Thus, this study was designed to measure the convergent validity of the three types of PM measures in both healthy adults and individuals with TBI. We aimed to investigate the convergent validity of the three types of tasks in two ways. First, we sought to investigate whether the PM deficits experienced by people with TBI are consistent across tasks. Second, we sought to examine the relationship between the three types of tasks. Results demonstrated that while all three types of measures were sensitive to PM deficits in TBI, there were differences in the aspects/processes of PM being measured. Data from the laboratory measure suggested a specific difficulty with detecting the correct cue. Data from the clinical measure suggested that TBI has a greater effect on time-based cues than eventbased cues and that the primary deficit is a prospective intention retrieval deficit rather than the retrospective memory component. In addition, those with TBI did not differ from healthy adults when the time delay was short enough, suggesting that PM is not universally impaired. Data from the self-report questionnaire suggested that those with TBI are more sensitive to difficulties with basic activities of daily living rather than instrumental activities on daily living. These results are discussed in terms of rehabilitation techniques that could focus first on cue detection and use basic activities of daily living as outcome measures.
\end{abstract}

Prospective memory (PM), remembering to remember and carry out a future action, is an important part of everyday life (Ellis \& Freeman, 2008; Rendell \& Thomson, 1999). For example, remembering to take out the trash or take medications at the correct time are both everyday PM tasks. For individuals with neurological disorders, including traumatic brain injury (TBI), schizophrenia, and Parkinson's disease, PM deficits have been shown to impact daily functioning (Mathias \& Mansfield, 2005; Raskin, 2009; Raskin, Buckheit, \& Waxman, 2012; Raskin et al., 2014; Shum, Levin, \& Chan, 2011; Shum, Ungvari, Tang, \& Leung, 2004; Shum, Valentine, \& Cutmore, 1999).

PM has been conceptualized using two current theories. The multiprocess view of PM suggests that PM performance can either be spontaneous or rely on strategic monitoring, depending on the nature of the task. Differences can occur due to disparities in type of task, type of cue, the nature of the ongoing task, and the individual. Thus, for example, tasks that must be remembered at a particular time have been suggested to require more strategic monitoring than those that must occur in response to an external cue (e.g., Einstein, McDaniel, Richardson, Guynn, \& Cunfer, 1995). More recently, Scullin, McDaniel, and Shelton (2013) expanded this model to suggest there is a dynamic interplay between the spontaneous retrieval and the strategic monitoring, such that individuals will monitor in a context where cues are expected but then disengage from monitoring when cues are not expected. They refer to this as the 
dynamic multiprocess framework. The preparatory attentional and memory processes (PAM) theory, on the other hand, asserts that PM always requires attentional processes that are resource demanding due to explicit monitoring or the need to maintain the intention (Smith, 2003). This is assumed to be the PM aspect of the task whereas the recall of what one was supposed to do is based on retrospective memory (RM). Given the prevalence of attention deficits in populations such as those with TBI, and the importance of PM performance to every- day life, investigating whether some aspects of PM might be spared could lead to important directions in management post TBI.

Measures of PM all have some common elements. These include the encoding and formation of an intention to be performed in the future, an ongoing task during the delay period, and a cue to signal it is time to perform the intention. Research on this topic has been performed using laboratory, selfreport, and clinical measures. It is thought that laboratory measures pro- vide greater ability to control and manipulate specific variables to answer particular theoretical questions (e.g., Einstein \& McDaniel, 2005). Self-report measures provide data on the subjective experience of PM (Roche, Moody, Szabo, Fleming, \& Shum, 2007) whereas clinical measures have been used in attempts to approximate more real-world applications of PM, such as calling the experimenter at a specified time (Rendell \& Thomson, 1999). Each of these measures provides valuable data on PM performance, but clear and consistent relationships between them have yet to be defined (e.g., Raskin \& Sohlberg, 2009; Rendell \& Thomson, 1999; Uttl \& Kibreab, 2011). Given the possibility that these measures are all capturing unique and not entirely overlapping aspects of PM, it is important to compare the findings from each.

Einstein and McDaniel (1990) introduced a dual- task laboratory paradigm that has been widely used in the literature. Like all PM tasks, there is a prospective remembering task embedded in an ongoing task, followed by a delay period. After this period, the ongoing task is reintroduced without a reminder of the PM task. The PM task, embedded in the ongoing task, is presented, and the response of the participant is measured (Einstein \& McDaniel, 2005). This laboratory paradigm has proven invaluable in testing specific theoretical questions about PM performance. However, these paradigms tend to involve only one type of PM response performed repeatedly, and the ecological validity is considered low (Delprado et al., 2012). In addition, the sensitivity of these paradigms to neurological disorders appears to vary with the characteristics of the PM and ongoing tasks (e.g., Foster, McDaniel, Repovs, \& Hershey, 2009).

There are also a number of currently used self-report PM measures, including the Comprehensive Assessment of Prospective Memory (CAPM; Chau, Lee, Fleming, Roche, \& Shum, 2007), the Prospective and Retrospective Memory Questionnaire (PRMQ, Crawford, Smith, Maylor, Della Sala, \& Logie, 2003), and the Prospective Memory Questionnaire (Hannon, Adams, Harrington, Fries-Dias, \& Gipson, 1995). Essentially, these measures all quantify common PM tasks that are undertaken in daily life and as suchmay have a higher personal significance then the other measures. Questionnaires have arguably high ecological validity and allow for a greater understanding of the experience of PM failures in daily life. They are gen- erally brief, easy to use and understand, and time- efficient (Man, Fleming, Hohaus, \& Shum, 2011). However, they have not been well correlated with other measures of PM, perhaps due to limited insight or awareness in individuals with brain injury (Fleming et al., 2009; Roche, Fleming, \& Shum, 2002).

The CAPM is one questionnaire that was designed to measure PM failures. It has been demonstrated to have good reliability (Chau et al., 2007). The full CAPM contains three sections: one that measures frequency of PM failure, one that measures the perceived importance of these failures, and the third that measures the perceived reasons for forgetting. It can be completed by an individual or by a significant other. A principal component analysis of the first part, Section A, revealed that there were two components, one that related to basic activities of daily living (BADL), such as daily self-care, and one that related to instrumental activities of daily living (IADL), such as household management activities (Chau et al., 2007).

To our knowledge, there are currently four standardized clinical measures of PM, and these are considered more naturalistic than laboratory measures. These are the Memory for Intentions Test (MIST; Raskin, Buckheit, \& Sherrod, 2010), the Cambridge Assessment of Prospective Memory Test (CAMPROMPT; Wilson et al., 2005), Virtual Week (Rendell \& Henry, 2009), and the Royal Prince Alfred Memory Test (Radford, Lah, Say, \& Miller, 2011). These tests have all shown good psycho- metric 
properties and sensitivity to neurological disorders.

Of these, the MIST has the ability to separate out different aspects of performance, such as type of cue and time delay. The MIST includes both time-based and event-based cues that are either two minutes or 15 minutes in duration between encoding and performance. The time-based cues allow for self-initiated retrieval ("In fifteen minutes, tell me that it is time to take a break"), and, unlike most laboratory tasks, the event-based cues are related to the events that need to be performed (i.e., "When I hand you a red pen, sign your name on the paper"). The response of participants can either be an action or a verbal response. Due to the MIST's ability to measure different attributes of PM, it also allows for six types of errors to be analyzed if failure of a PM task were to occur (Raskin, 2009). The MIST has been demonstrated to be sensitive to PM deficits in individuals with TBI (Raskin, 2009), Parkinson's disease (Raskin et al., 2011), HIV (e.g., Woods, Weber, Cameron, Dawson, \& Grant, 2010), and schizophrenia (Raskin et al., 2014; Twamley et al., 2008) and has been shown to have good psycho- metric properties (Raskin, 2009; Woods et al., 2008).

Previous studies comparing questionnaires and clinical measures have yielded mixed results. One previous study found no correlation between the CAPM and total score on the MIST in individuals with TBI, suggesting either that the two measures are measuring separate functions or that subjective experience and objective performance are not the same (Fleming et al., 2009). Relatives' report on frequency of PM fail- ure did correlate with the MIST total score as well as both the time-based cue and event-based cue scales, which suggest that the difference may be due to poor subjective awareness. No data were provided on the relationship between the CAPM and the 24-hour delay item on the MIST. Another study of people with TBI used the PRMQ and found that while the MIST did not correlate with responses on the self- report questionnaire, the MIST did correlate with performance in daily life on 10 items over a week as reported by a significant other (Raskin \& Sohlberg, 2009). In a study of healthy older adults the MIST was uniquely related to the IADL component of the PRMQ (Woods, Weinbom, Velnoweth, Rooney, \& Bucks, 2012). In studies that compared a laboratory test and self-reported questionnaires, it was also reported that performance on the laboratory test correlated with the informant-reported score (Chi et al., 2014) but not with the self-reported score in older adults (Chi et al., 2014) or in those with Parkinson's disease (Foster et al., 2009). Thus, this again suggests that the questionnaires are tapping into an aspect of the individual's perception of his or her performance that may not be related to the individual's actual performance.

It seems possible, and even likely, that these three types of measures are sampling different attributes of PM. For example, it has been argued that the cognitive demands in daily life, as measured by a questionnaire, may be very different than those in a laboratory setting (Fleming, Doig, \& Katz, 2000). As has been suggested, different PM tasks could very well challenge different cognitive systems depending on the task (McDaniel \& Einstein, 2011). In fact, there is growing evidence that different types of PM tasks rely on different brain systems. For example, tasks with focal cues may require little strategic monitoring and thus rely on the medial temporal structures while those with nonfocal cues may require activity of the prefrontal cortex (e.g., McDaniel \& Einstein, 2011).

These tasks may also require different levels of attentional resources. The laboratory measures tend to use only event-based cues, thus they may be less attention demanding than the time-based tasks in the MIST. Furthermore, unlike the MIST, the event-based cue in the laboratory measure is usually embedded within the ongoing task, which may further serve to focus attention on the cue. On the other hand, in the laboratory paradigm the embedded PM task (i.e., press a key when you see a specific word) does not explicitly put the participant in a "retrieval mode"requires participants to enter this mode on their own. In contrast, the event cues (and not the time cues) of the MIST puts participants in a retrieval mode (i.e., by handing the participants a form requesting medical records) in which they may be aware something should happen because of the high association between the intended action and the cue. On the other hand, the CAPM queries performance on both event-based (e.g., forgetting to lock the door when leaving home) and timebased (e.g., forgetting to take tablets at a pre- scribed time) everyday tasks. It can be argued that the CAPM is the only one that measures tasks in which the individual must form his or her own intention and create his or her own plan for execution (e.g., Dobbs \& Reeves, 1996). For the other 
tasks this is provided for the participants by the experimenter. However, because the CAPM relies on retrospective subjective report, it is not known whether it is an accurate reflection of performance.

Differences between the measures may shed light on research findings based on clinical populations, and, therefore, it would be important to examine the convergent validity of these measures not only on healthy populations but on those with PM deficits. Research on TBI has revealed significant deficits in PM performance, presumably due to impairment of the prefrontal cortical regions, and individuals with specific frontal lobe damage have shown impairments in PM (Neulinger, Oram, Tinson, O'Gorman, \& Shum, 2015). These individuals show deficits in the total score on the MIST as well as differences in performance on the 2- and 15-min time delays (Raskin, 2009). Adults with TBI, like healthy adults, are better at event-based cues than at time-based cues (Mioni, Rendell, Henry, Cantagallo, \& Stablum, 2013; Raskin, 2009). Studies on participants with TBI using the laboratory paradigms have also shown difference in performance. In a study done by Shum et al. (1999) using time-, event-, and activity-based PM tasks, the results revealed differences in all three types of tasks, with the individuals with TBI performing significantly worse on all tasks (Shum et al., 1999). The CAPM has been used in previous studies with individuals who have TBI and has demonstrated sensitivity to perceived failures in this population (Huang et al., 2014) and has been analyzed according to Ellis's (1996) five phases of prospective memory to demonstrate that those with TBI had difficulty with encoding and formation and initiation of prospective memories (Roche et al., 2007). In reviewing the literature, however, we are not aware of a prior study that used all three types of measures in a single study. Thus, it would be worth- while to measure all three of them within the same population. In the first place, this would allow for a more comprehensive evaluation of both laboratory- based and naturalistic PM functioning. Second, it would allow for an analysis of the specific aspects of PM being measured by each task.

This study compared performance of healthy adults and individuals with TBI on the MIST (Raskin, 2009), an Einstein and McDaniel dual-task laboratory paradigm that was previously published by Pereira, Ellis, and Freeman (2012), and the CAPM (Chau et al., 2007). The primary aim of the study was to understand how individuals with TBI perform on each measure given concurrently in order to measure the convergent validity of these tasks in this population so that it could be determined whether deficits seen in individuals with TBI are consistent across the different tasks. The secondary aim was to examine the association between the tasks. Convergent validity was evaluated in both healthy individuals and those with TBI because TBI is a widely studied diagnostic group and the group for whom the most attention has been paid in the literature on PM rehabilitation (e.g., Raskin, Smith, Mills, Pedro, \& Zamroziewicz, 2017). Our main hypothesis was that the three measures would show some overlap but that there would be significant aspects of PM measured uniquely by each measure impaired in TBI, a Stroop Color-Word Interference task was administered within the laboratory measure to evaluate the relationship between aspects of poor executive functioning measured by the Stroop on PM performance.

Method

\section{Participants}

One hundred healthy adults (HA; 51 females) and 50 people with TBI (18 females) took part in this study. Demographic information for the two groups is pre- sented in Table 1. Student's $t$ tests and $\chi^{2}$ analyses revealed no significant differences between the groups on any demographic variables.

\section{Inclusion criteria}

All participants spoke English as a primary language. Individuals with TBI were at least one year post injury, had lowest postresuscitation Glasgow Coma Score (Teasdale \& Jennett, 1974) of 8-12, and had obtainable medical records. People with TBI were recruited through local hospitals and the local brain injury asso- ciation in the US. Healthy adults were relatives of the people with brain injury, employees of Trinity College, or members of the community. Descriptive injury- related information of the TBI group is presented in Table 2.

\section{Exclusion criteria}


Any potential participant that had previous neurologi- cal or psychiatric illness, history of substance abuse or diagnosed learning disability, visual impairment that would interfere with reading the test materials, and/or experienced seizure during the previous year was

Table 1. Demographic information for the two groups.

\begin{tabular}{lccrl}
\multicolumn{4}{c}{ Healthy adult } & Traumatic brain injury \\
& $(N=100)$ & $(N=50)$ & Group differences \\
\hline Age: Mean & $29.78(11.27)$ & $31.54(16.56)$ & $t=1.03, \quad p=.61$ \\
Education: Mean & $14.41(1.30)$ & $15.13(2.43)$ & $t=1.11, \quad p=.54$ \\
Handedness & $83 \mathrm{R} \mathrm{17L}$ & $39 \mathrm{R} 11 \mathrm{~L}$ & $X^{2}=0.45, \quad p=.20$ \\
Sex & $51 \mathrm{~F} 49 \mathrm{M}$ & $32 \mathrm{M} \mathrm{18F}$ & $\mathrm{X}^{2}=0.71, \quad p=.17$ \\
\hline
\end{tabular}

Note. Age and education in years. Standard deviations in parentheses. $\mathrm{R}=$ right; $\mathrm{L}=$ left.:

Our secondary hypothesis was that the measures would show specificity to the deficits demonstrated by individuals with TBI. The Pereira et al. (2012) task also allows for an analysis of the effect of cueaction relatedness. The results of this study would provide insight as to whether the three measures are measuring the same or different aspects of PM. In addition, given the assumption that PM is mediated by prefrontal cortical regions and the likelihood that these regions are excluded from the study. No individual with TBI had undergone cognitive rehabilitation.

Table 2. Injury information for the group with TBI.

\begin{tabular}{lcr} 
& $M(S D)$ & Range \\
\hline GCS at scene or ED & $11.87(3.88)$ & $8-15$ \\
Post traumatic amnesia (days) & $1.35(6.45)$ & $0-9$ \\
Loss of consciousness (days) & $11.23(19.66)$ & $0-33$ \\
Time post TBI (months) & $29.32(12.45)$ & $12-60$ \\
\hline
\end{tabular}

Note. TBI = traumatic brain injury; GCS = Glasgow Coma Scale.:

\section{Screening measures}

All participants were administered the Brief Psychiatric Rating Scale (Overall \& Gorham, 1962) to screen for psychiatric illness. No individual with severe depression ( $\geq 21$ on the Beck Depression Inventory; Beck \& Steer, 1987) anxiety ( $\geq 30$ on the Beck Anxiety Inventory; Beck \& Steer, 1990), or global cognitive dysfunction (severe impairment on four or more of the subscales of the Neurobehavioral Cognitive Status Examination; Kiernan, Mueller, Langston, \& Van Dyke, 1987) was included.

\section{Measures}

The Memory for Intentions Test (MIST)

The MIST (Raskin et al., 2010) is a 30-min, eight-trial test during which participants engage in a word search puzzle as the ongoing task. A complete description of the MIST administration and scoring procedures can be found in Raskin (2009). Briefly, it is composed of four trials with event-based cues (e.g., "When I hand you a postcard, self-address it"). and four trials with time-based cues (e.g., "In 15 minutes, tell me it is time to take a break"), with each item scored from $0-2$ points; thus, the separate event-based and time-based scales have scores ranging from 0 to 8 . The time- and event-based trials are balanced for delay interval (i.e., 2- and 15-min delay periods) and response modality (i.e., verbal and action responses). The MIST allows for separate scoring of time-based trials (8 points possible), eventbased trials ( 8 points possible), 2 -min delay periods ( 8 points possible), 15 -min delay periods ( 8 points possible), verbal response trials ( 8 points possible), and action response trials ( 8 points possible), which are summed for a total of 48 possible points. However, this involves inclusion of the score of each trial three times in the total score (e.g., Trial 1 is a 2-min delay trial, time-based cue, and verbal response, thus contributing to the 2-min delay, time-based cue, and verbal response scores). A large digital clock is in full view of the participant at all times. For the event-based trials, the cues all have high cue-action 
relatedness and were considered to be ecologically relevant, meaning they are related to the response required and could naturally elicit that required response (e.g., When I hand you a reque st for records form, please write your doctors' names on it). The ongoing task is nonfocal as the word search is not related to the PM items. Prior studies support the reliability (Raskin, 2009; Woods et al., 2008) and construct validity (e.g., Raskin et al., 2010; Woods et al., 2009) of the MIST.

At the completion of the eight MIST trials, participants are given eight multiple-choice recognition items (e.g., "At any time during this test, were you supposed to: 1) tell me to make an appoin tment; 2) tell me when I can call you tomorrow; 3) tell me to call for a prescription."). The recognition scale is included as a way to determine whether PM failures are due to encoding versus retrieval failures. Impairment on recognition items is likely to reflect deficits in RM rather than PM functions. Furthermore, a 24-hour delay trial was administered, for which examinees were instructed to leave a voicemail message for the examiner the day after the exam indicating the number of hours the participant slept the night after the evaluation. In addition, the following error types were coded:

(a) no response (i.e., response omission errors); (b) task substitutions (e.g., replacement of a verbal response with an action or vice versa); (c) loss of content (e.g., acknowledgment that a response is required to a cue, but failure to recall the content); and (d) loss of time (i.e., performance of an intention greater than $\pm 15 \%$ before or after the target cue). If the participant makes no response to the PM cue, those are coded as "no response" errors and are presumed to be directly due to failure of PM (i.e., cue detection). Task substitution errors (e.g., intrusions and perseverations) are likely multidetermined, but presumed to be due to executive control deficits (e.g., Carey et al., 2004). Loss of content errors most likely reflect RM failures, and loss of time errors seem to be due to difficulty with strategic monitoring or timing.

\section{The Comprehensive Assessment of Prospective Memory (CAPM)}

The CAPM is a self-report questionnaire used to assess PM. It takes approximately $10-15 \min$ to complete. The CAPM contains three sections: The first section measures the frequency of PM failure, the second determines the perceived importance of such failures, and the third gauges the perceived reasons for prospective remembering and forgetting. In this study we used only Section A containing 39 items relating to frequency of PM failure in the last month, as has been done in previous studies of TBI (Fleming et al., 2009). Items are rated on a 5-point scale. This scale indicates that $1=$ "never," $2=$ "rarely," 3 = "occasionally," 4 = "often," and 5 = "very often."

Most items in Section A can be categorized into one of two subscales, IADL and BADL, established by Waugh (1999) using a principal component analysis. There are 23 items relating to IADL, such as "Leaving the iron on" and "Not remembering to pay bills." For the BADL subscale there are 10 items such as "Not locking the door when leaving home" and "Leaving water taps on." Given the "not applicable" category allowed, total scores and subscale scores were not used. Instead, for each participant three scores were calculated (total CAPM, IADL subscale, and BADL subscale) by summing the participant's ratings on the 1-5 scale for all completed items and dividing by the total number of items less the not applicable items. Therefore, the possible range for mean total and subscale CAPM scores was 1-5, with higher scores indicat-ing more frequently perceived PM failure.

\section{Laboratory PM task (Pereira et al., 2012)}

The experimental session involved a practice phase for the ongoing task, followed by instructions for the PM task, a filled delay period, and the main ongoing plus PM task. The effects of task variables on PM performance were examined on two measures: performance accuracy and latency for responding to the PM cues. Additionally, performance accuracy on the ongoing task and response latency to non-PM cue items were recorded.

A simple computer-based activity in which participants had to sort 20 different noun words into two different categories (natural vs. manmade) was pre- pared for the practice phase. For the PM cue-action pairings, two lists of six noun-verb pairs were com- piled: One list comprised six related noun-verb pairs and the other six unrelated pairs. For the related list, noun-action words that had a mild semantic association (Forward Strength (FSG) < 0.1; Nelson, McEvoy, \& Schreiber, 1998) were selected. In the unrelated list, the nouns from the related list were reassembled with the verbs to create new pairs with no obvious associative relation between them. The word pairs had normative medium values of familiarity (range $=3.71-4.59$ on a scale of 1 to 7 ) and memorability (range $=3.71$ to 3.34 on a scale of 1 to 7; Molander \& Arar, 1998). For the main ongoing task, a set of 100 nouns (94 
new and 6 cue-words) was created.

Participants were tested individually. They were informed that the session started with a practice task involving a simple computer-based activity in which they would have to allocate 20 different words into one of two different categories, natural or manmade, by pressing the appropriate allocated key. Items remained on screen until the participant produced a response. This was followed by instructions for the PM task. Participants were presented with a set of six cue-action word pairs to learn. Half of the participants were presented six semantically mildly associated cue-action pairs. The other half of the participants were presented six cue- words that were not semantically associated in any obvious way. The mildly associated cue-action word pairs were as follows: ball-throw; coat-hang; flower-smell; lemon-squeeze; needle-prick; pencil-sharpen. All participants were informed that they would later perform a word-sorting task similar to the one performed during the practice phase. They were told that they would see a fixation in the center of the computer screen for $3 \mathrm{~s}$ and that this would be followed by a sequence of words presented one at a time. They were asked to report, by pressing the relevant computer key, whether the words belonged to the category "manmade," or to the category "natural." They were also told that some of the words of the task would have been presented in the previously encoded (prospective) set while others would be new. More specifically, participants were told that upon seeing the previously presented words (PM cue) they would have to press the computer key with the purple dot on it. After this, they would have to continue the word-sorting task by pressing the appropriate key determining whether that would be a natural or manmade stimulus.

Following presentation of all six items, participants were asked to complete a computerized version of the Stroop color-word interference task. This task required them to press a key on a response pad with a sticker corresponding to the color of the ink of the word displayed on the screen (red, yellow, blue, or green). This lasted approximately 5 min.

Instructions for the main word-sorting (ongoing) task were then provided. However, none of the previously given information for the prospective task was given at that moment; if participants asked for more information about these previously given instructions at this point, they were simply told to do as they thought best.

Participants were reminded that they would see a fixation in the center of the computer screen for 3 seconds and that this would be followed by a sequence of words. They were asked to decide, by pressing the relevant computer key, whether the words belonged to the category "man- made" (the computer key "z" had a sticker that said "man- made") or to the category "natural" (the computer key "/" had a sticker that said "natural"). The 100 words (94 new, 6 PM cues) of the word-sorting task were then presented. Items remained on screen until the participant produced a response that he or she considered to be appropriate, by pressing a computer key. In the set of 100 words the cues were presented in the 8th, 20th, 44th, 55th, 82nd, and 99th position. These positions were not completely randomly selected because it was necessary to ensure that they were relatively evenly spread across the set of 100 words in such a way that the participant could not easily anticipate the exact position in which the cue would appear.

On completion of the word-sorting task, participants were asked whether they remembered the instructions that had been given to them. This included describing what they thought they had been asked to do and also to recall all the six cue-action word pairs. As Maylor, Smith, Della Sala, and Logie (2002) recommended, while studying prospective remembering it is crucial to separate PM failures from RM ones. Participants may perform poorly in a PM task not necessarily as a result of a PM failure, but because of a RM failure. Therefore, in the present study, participants were questioned at the end of the testing to ensure that they had successfully retained the PM instructions.

\section{Procedure}

All participants were tested individually after obtaining informed consent. Total testing time was approximately 2 hours. Breaks were given if requested or if the participant complained of fatigue. The order of administration of the three tests was counterbalanced across participants.

\section{Data analysis}

The groups were compared on demographic variables using Student's $t$ tests and $\chi^{2}$ as appropriate. None of the MIST measures were normally distributed as revealed by a series of Shapiro-Wilk $W$ tests (all $p$ s < .01). Thus a series of nonparametric tests were used to compare healthy adult and TBI samples on variables from the MIST. In all cases reported analysis of variance (ANOVA) results on raw data were 
confirmed with rank-transformed data; in no case was there a discrepancy in findings. Alpha was set at .05 , and all analyses were two-tailed. Normality tests were not necessary in order to conduct a nonparametric correlation analysis between MIST and the laboratory measure or MIST and CAPM, as normality was not an assumption. To measure convergent validity, Spearman rank order correlations were used to examine the relationship between the MIST, the CAPM, and the laboratory test. In order to compare those with high scores on the executive function measure and those with low scores, a median split was performed, and Student's $t$-tests were used to compare the groups.

\section{Results}

\section{MIST performance}

Descriptive data are presented in Table 3. A repeated measures ANOVA for Time Delay (2 min, $15 \mathrm{~min}$ ) $\times$ Group $(\mathrm{HA}, \mathrm{TBI})$ revealed a significant main effect for time delay, $F(1,143)=85.70, p<.001, \eta^{2}=.39$, such that performance was superior at 2-min delays compared to 15-min delays; the main effect for group was also significant, $F(1,143)=44.26, p<.001, \eta^{2}=.25$, such that healthy adults showed superior performance to those with brain injury; and the interaction was significant indicating that the effect of time was more pronounced for individuals with TBI, $F(1,143)=11.89, p<.01, \eta^{2}=.079$.

For Type of Cue (event, time) $\times$ Group (HA, TBI), the main effect for type of cue was also significant, $F(1,145)=89.36, p<.001, \eta^{2}=.40$, such that performance for event-based cues was superior to performance for time-based cues overall. The main effect for group was significant, $F(1$, $145)=53.40, p<.001, \eta^{2}=.29$. The interaction was also significant indicating that the effect of cue was greater for individuals with TBI, $F(1,145)=8.98, p<.01, \eta^{2}=.06$.

For Type of Response (action, verbal) $\times$ Group (HA,TBI), the main effect of type of response was significant, $F(1,143)=17.61, p<.001, \eta^{2}=.12$, such that performance on verbal response tasks was superior to that on action response tasks. The main effect of group was significant, with the HA group performance superior to that of the TBI group, $F(1,143)=44.60, p<.001, \eta^{2}=.25$. The interaction was significant, indicating that the effect of response was greater for the HA group, $F(1,145)=89.2, p<.01$, $\eta^{2}=.03$.

Individuals with TBI had significantly more PM (no response) errors, indicating no recall of the need to perform an intention, $t(149)=10.21, p<.01, d=1.67$. They also performed significantly more poorly on the recognition items, indicating that $\mathrm{RM}$ is also impaired, $t(149)=12.33, p<.01, d=2.02$. On the more naturalistic 24-hour recall task, there was no difference between the groups. See Table 3 for the performance of the two groups on the MIST.

Table 3. Performance of the two groups on the MIST.

\begin{tabular}{lcccc}
\hline & $\begin{array}{c}\text { Healthy adult } \\
(N=100) M\end{array}$ & $\begin{array}{c}\text { Traumatic brain } \\
(N=50) M(S D)\end{array}$ & $t(148)$ & $d$ \\
\hline Delay: 2 minutes & $7.80(0.75)$ & $6.94(1.60)$ & 2.82 & 0.46 \\
Delay: 15 minutes & $6.90(1.30)$ & $5.00(2.12)$ & $12.21^{* *}$ & 2.00 \\
Event cues & $7.75(0.65)$ & $6.71(1.36)$ & $6.95^{* *}$ & 2.07 \\
Time cues & $6.99(1.11)$ & $5.24(1.71)$ & $10.40^{* *}$ & 1.13 \\
Action response & $7.28(0.97)$ & $5.76(1.95)$ & 3.17 & 1.70 \\
Verbal response & $7.49(0.72)$ & $6.41(1.33)$ & $13.91^{* *}$ & 0.51 \\
Total correct & $44.15(4.43)$ & $35.82(9.50)$ & $18.46^{* *}$ & 2.28 \\
PM errors & $0.14(0.54)$ & $1.83(0.98)$ & $41.53^{* *}$ & 3.02 \\
Recognition & $7.68(0.65)$ & $7.00(1.03)$ & 1.71 & 6.80 \\
24-hour recall & $0.76(0.93)$ & $0.50(0.82)$ & 0.28 \\
\hline
\end{tabular}

Note. MIST $=$ Memory for Intentions Test $\mathrm{PM}=$ prospective memory.

$* * p<.01 . * * * p<.001$.

\section{CAPM performance}

For each participant, the average rating for items in both subscales (IADL and BADL) as well as for all 39 items (total CAPM) was calculated. The averaging of scores on the three scales was to take into account missing responses due to items being "not applicable." Mean ratings and standard deviations for the items in the IADL and BADL subscales and total CAPM were calculated. On the CAPM, individuals with TBI indicated 
significantly lower BADL performance in daily life than controls, $t(149)=4.17, p<.05, d=0.68$, but there was no difference between the groups in terms of IADL or total performance. See Table 4.

\section{Laboratory PM measure performance}

The laboratory PM measure data were analyzed using a $2 \times 2$ ANOVA of Group (HA, TBI) $\times$ Cue-Action Relatedness (related, unrelated). There was a significant main effect of cue-action relatedness, $F(1,141)=$ 19.20, $p<.01, \eta^{2}=.14$, such that PM performance was better overall when the cue was semantically related to the action. There was also a significant main effect of group, with HA individuals demonstrating significantly better performance than individuals with TBI, $F(1,141)=22.19, p<.01, \eta^{2}=.18$. The interaction was not significant. See Table 5 for performance data on the two groups on this measure. The proportion of cueaction word pairs that were remembered accurately at the end of the task was also calculated as a measure of retrospective recall. The number of correctly recalled cue-action word pairs was calculated, and there was no effect of cue-action relatedness on the recall of the six word pairs. However, recall was impaired in the group with TBI compared to the group of HA, $t(149)=9.79, p<.01, d=1.60$.

\section{Convergent validity of the different measures}

Convergent validity was measured between the MIST and the laboratory measure, using Spearman rankorder correlations. For individuals with TBI, total cor- rect on the laboratory task correlated significantly with a number of measures on the MIST. This included total correct and PM errors. See Table 6. For the HA, the only significant correlation between the MIST and the laboratory measure was MIST recognition and total correct on the laboratory measure, $r(98)=.339, p<.05$. In order to measure the convergent validity between the MIST and the CAPM Spearman rank-order correlations were used. For the individuals with TBI, there were no significant correlations between the CAPM and either the MIST or the laboratory measure. For the HA, the MIST total errors correlated significantly with BADL on the CAPM, $r(98)=.211, p<.05$, but there were no significant correlations between the CAPM and the laboratory measure.

Table 4. Performance of the two groups on the CAPM.

\begin{tabular}{llclc}
\hline & $\begin{array}{l}\text { Healthy control } \\
(\mathrm{N}=100) \mathrm{M}(\mathrm{SD})\end{array}$ & $\begin{array}{l}\text { Traumatic brain injury } \\
(\mathrm{N}=50) \mathrm{M}(\mathrm{SD})\end{array}$ & $\mathrm{t}(148)$ & $\mathrm{d}$ \\
\hline Basic ADL & $1.54(0.36)$ & $1.80(0.23)$ & $4.17 *$ & 0.68 \\
Instrumental ADL & $1.96(0.48)$ & $1.94(0.51)$ & 1.02 & 0.17 \\
Total score on CAPM & $1.83(0.41)$ & $1.81(0.45)$ & 1.43 & 0.23 \\
\hline
\end{tabular}

Note. $\mathrm{CAPM}=$ Comprehensive Assessment of Prospective Memory; $\mathrm{ADL}=$ activities of daily living. $* p<.05$.

Table 5. Performance of the two groups on the laboratory measure.

\begin{tabular}{lcccc}
\hline & $\begin{array}{c}\text { Healthy control } \\
(N=100) M\end{array}$ & $\begin{array}{c}\text { Traumatic brain } \\
\text { injury }(\mathrm{N}=50) M\end{array}$ & $t(148)$ & $d$ \\
\hline Correct (max. 12) & 6.42 & 4.07 & 3.64 & 0.60 \\
False positives & 0.26 & 0.50 & $12.04^{* *}$ & 1.97 \\
Correct (related)/proportion correct & 0.81 & 0.71 & $7.12^{*}$ & 1.18 \\
Correct (unrelated)/proportion correct & 0.52 & 0.42 & $10.87^{* *}$ & 1.78 \\
Retrospective recall & 7.77 & 6.21 & $8.29^{*}$ & 1.36 \\
\hline
\end{tabular}

$* p<.05 . * * p<.01 . * * * p<.001$. 
Table 6. Correlations between the MIST and the laboratory measures for the individuals with brain injury.

\begin{tabular}{lcccc}
\hline & $\begin{array}{c}\text { Lab } \\
\text { measure }\end{array}$ & $\begin{array}{c}\text { Lab } \\
\text { measure }\end{array}$ & $\begin{array}{c}\text { Correct } \\
\text { (related }\end{array}$ & $\begin{array}{c}\text { Retrospective } \\
\text { recall }\end{array}$ \\
\hline MIST time cues & $.641^{* *}$ & .038 & .168 & .041 \\
MIST event cues & $.564 * *$ & $.219 *$ & .345 & .096 \\
MIST total correct & $.628^{* *}$ & -.250 & .047 & .220 \\
MIST recognition & $.608^{* *}$ & -.451 & -.174 & $.331^{*}$ \\
MIST PM errors & $-.446^{* *}$ & -.115 & -.151 & -.132 \\
MIST total errors & $-.702 * *$ & $.442^{*}$ & -.068 \\
\hline
\end{tabular}

Note. MIST = Memory for Intentions Test; $\mathrm{PM}=$ prospective memory. Correlations are $r$ values.

$* p<.05 . * * p<.01$.

Table 7. Comparison of the TBI group with low performance on the Stroop to the TBI group with high performance on the Stroop.

\begin{tabular}{|c|c|c|c|}
\hline & Stroop $\leq 20$ & Stroop $>20$ & $t(48)$ \\
\hline MIST total correct & $0.91(0.88)$ & $1.82(1.08)$ & $12.17 * * *$ \\
\hline MIST PM errors & $0.00(0.00)$ & $0.43(0.53)$ & $7.84 * *$ \\
\hline MIST recognition & $7.83(0.45)$ & $6.91(1.05)$ & $9.50 * *$ \\
\hline MIST 24-hour recall & $0.42(0.76)$ & $0.00(0.00)$ & $17.41 * * *$ \\
\hline BADL & $1.52(0.32)$ & $1.34(0.34)$ & 2.33 \\
\hline IADL & $1.93(0.44)$ & $1.67(0.49)$ & 3.94 \\
\hline Total CAPM & $1.66(0.49)$ & $1.83(0.36)$ & 1.61 \\
\hline Correct on laboratory task & $7.51(3.96)$ & $5.92(4.22)$ & 1.33 \\
\hline False positives on laboratory task & $0.28(0.52)$ & $1.09(1.30)$ & $9.16 * *$ \\
\hline
\end{tabular}

Note $\mathrm{TBI}=$ traumatic brain injury; MIST $=$ Memory for Intentions Test; $\mathrm{PM}=$ prospective memory; $\mathrm{BADL}=$ basic activities of daily living; IADL = instrumental activities of daily living; CAPM = Comprehensive Assessment of Prospective Memory.

$* * p<.01 . * * * p<.001$. 
When the TBI individuals were separated into low and high performance on the Stroop ColorWord Interference Test, using a median split, the groups differed in false positives on the laboratory measure, the MIST total correct, MIST recognition, and PM errors but not the CAPM. See Table 7.

\section{Discussion}

Overall, results of this study support previous research that has demonstrated that individuals with TBI are impaired compared to HA in PM performance and report significantly more PM deficits than HA (see Shum et al., 2011, for a review). More specifically, these results suggest that the impairment is most apparent when time delays exceed working memory time span, self-initiated retrieval is required, and the response requires an action. There is also a loss of RM content, most likely due to an independent RM impairment as seen on the recognition tasks, although an error analysis from the MIST suggests that the PM impairment is considerably more prevalent than the RM impairment. Both groups showed a superiority for recognition over recall but the individuals with TBI were impaired in recognition, thus suggesting a deficit in both PM and RM systems. This is consistent with previous studies that have demonstrated separate significant effects in individuals with TBI (Pavawalla, Schmitter-Edgecombe, \& Smith, 2012; Raskin et al., 2011). The lack of a deficit in the TBI group when the delay period was short suggests that these data are more consistent with the multiprocess theory than the PAM theory. In other words, under some conditions, individuals with TBI are able to perform adequately despite overall PM deficits. The finding of a better performance on event-based than on timebased tasks is consistent with previous findings in populations with neurologic impairments (e.g., Raskin et al., 2012; Raskin et al., 2011). This has generally been explained by assuming that time-based tasks require more self-initiated retrieval and therefore greater cognitive resources allocation (e.g., Kvavilashvili, Cockburn, \& Kornbrot, 2012), although some research has suggested that this may have to do with the extent to which the ongoing task and PM task share the same central executive resources (d'Ydewalle, Bouckaert, \& Brunfaut, 2001).

Results from the laboratory measure suggest that some of the difficulty with PM performance may be due to responding to an incorrect cue. Although no options for this are included in the MIST, the relation- ship of low cognitive control (i.e., poor Stroop performance) with PM errors may suggest a similar underlying pathology. There is some evidence to sug-gest that one aspect of PM performance (such as recognition of the PM cue) involves lateral prefrontal cortex plus precuneus (Burgess, Scott, \& Frith, 2003), which may be the same regions as those that mediate top- down attentional processes used for the Stroop (Banich et al., 2000). This further lends support to the multi- process theory.

Results from the questionnaire suggest that the indivi duals with TBI perceived and reported greater difficulty with BADL activities than IADL activities, when compared to healthy participants. Both groups reported higher rates of IADL PM failures than BADL PM failures. However, when comparing the groups, the group with TBI was not different in their self-report from the healthy participants on IADL but reported a higher number of BADL PM failures than the healthy group. This may be because failures at this level more significantly impact independence and generally represent a greater level of impairment (e.g., Harris, Jette, Campion, \& Cleary, 1986; Mitchell et al., 2011). This sug- gests that rehabilitation efforts might start with BADL activities that are perceived as more likely to be impaired. However, it should be noted that whereas the ratings of IADL in this study are consistent with previous reports, the BADL ratings do appear to be higher than those in pre- vious studies (Fleming et al., 2009; Roche et al., 2002), and the finding of higher ratings of failures among people with TBI than healthy participants is also not consistent with previous findings (Huang et al., 2014). Thus it is unclear whether this suggests a difference between this TBI population and others in terms of either basic ADL functioning or self-awareness. Reports of poor self-awareness of memory changes in general, and PM deficits in particular, have been reported widely (e.g., Fleming, Strong, Ashton, 1996; Roche et al., 2002; Sherer, Boake, Levin, \& Silver, 1998). And, in fact, there have been previous reports of greater ratings of impairments on physical limitations, such as those most likely to be measured by a scale of basic ADL, as compared to cognitive changes (Sherer et al., 1998).

These results support previous findings that the similarity of the retrieval cue and the intended action can influence the likelihood of successful PM performance. We found this to be true with the laboratory measure with the healthy adults, as has been shown previously (Ellis, 1996; McDaniel, Guynn, Einstein, \& Breneiser, 2004; Pereira et al., 2012). We do not know of a previous study that 
has shown this same effect in individuals with TBI. This may point to a potential rehabilitation strategy for individuals with TBI in which individuals are trained to identify cues that are related to the intended action. In addition, this suggests that tests like the MIST, in which all event cues have highly related cue-action pairs, may be measuring only one aspect of performance on event-based cues.

When the groups are separated by adequate versus poor performance on a task of executive functioning, those with low executive functioning demonstrate significantly impaired performance on the clinical mea- sure and the false-positive response rate of the laboratory measure. Previous studies have not demonstrated a relationship between Stroop performance and PM in children with TBI (Ward, Shum, McKinlay, Baker, \& Wallace, 2007). Time-based PM has been shown to be related to Stroop performance in adults with TBI (Mioni, Stablum, McClintock, \& Cantagallo, 2012) but these data suggest that both time- and event- based PM rely on these executive abilities and are specifically related to PM errors. Rule monitoring was shown to be related to PM performance on the Rivermead Behavioral Memory task in individuals with TBI, which is similar to the MIST (Paxton \& Chiaravalloti, 2014). In contrast, these particular executive abilities do not seem to be related to the kind of PM required for the laboratory task or for awareness of deficits in daily life. This suggests that this aspect of executive functioning (error detection) may be related to some but not all features of PM.

Thus, these results support findings of separate contributions of different prefrontal regions to different aspects of PM performance (Burgess et al., 2003). The Stroop task has been previously shown to cause activation of anterior cingulate and inferior prefrontal regions (Leung, Skudlarski, Gatenby, Peterson, \& Gore, 2000). Therefore, the MIST may be tapping more into these functions whereas the laboratory task may be more related to cue detection and monitoring. In addition, responses on the questionnaire were not influenced by the aspect of executive functioning that is measured by the Stroop, and thus these questionnaires may be tapping into metacognitive awareness that is mediated by other executive functions. This study is unable to answer this question, and future studies that include imaging data on lesion location are necessary.

In terms of convergent validity, first comparing the clinical measure (MIST) to the laboratory measure, it is interesting that there was little relationship between these two forms of PM measurement for the HA but that there was a strong relationship for those with TBI. Perhaps the tasks, designed not to be too difficult for those with TBI, were too simple for the HA, although ceiling effects were not noted. It may be the case that those whose PMfunctions are intact are using a variety of processes towards success but that those who are experiencing impairments are all experiencing similar impairments. In other words, there may be many paths to success, so that the relationship between performances for those who are successful may not be consistent. On the other hand, for those withTBI, these data suggest that both tasks are tapping into similar or relatedimpairments that cause problems with successful performance on both the laboratory measure and the clinical measure, leading to a significant relationship. In addition, it was not surprising that the greatest relationship was with the event-based items on the clinical measure. This highlights the problem with using laboratory measures that include only event-based items if one is interested in a comprehensive picture of PM performance. The clinical measure was also related to falsepositive performance on the laboratory measure, in this case the number of PM errors. This may suggest that time-based remembering is related to self-monitoring more than event-based PM.

For the self-report measure, there was no relationship to either the laboratory measure or the clinical measure for individuals with brain injury. For those in the HA group, there was only a relationship between BADL on the CAPM and the MIST. It is not clear from this study why the questionnaire did not demonstrate a greater relationship to the other tasks. Perhaps this finding may be explained by the fact that the questionnaire is the only task that includes items requiring self-initiated planning. On the other hand, this finding is consistent with previous studies that have suggested that the issue of self-awareness is another factor that should be examined in more detail (Fleming et al., 2009). To clarify which of these is the likely explanation, future studies could take advantage of the significant other version of the CAPM. If significant correlations are found between those CAPM responses and the clinical or labora- tory performance of those with TBI, the absence of significant relationships in this study are most likely explained as self-awareness deficits. In addition, perhaps perfor- mance on the CAPM could be compared to a diary study of PM performance in daily life, as rated by a significant other. For the time being, it would be important to bear in mind the lack of a relationship between these measures when using only one or the other in future studies or clinical setting.

There are several limitations to this study. The first is the limitations of the singular measures given. For example, the laboratory measure includes only event-based items. Further the limitations of the MIST 
include less precise control of participant input than the computerized laboratory measure. The MIST may also not be measuring the same types of PM problems as are measured in daily life by the CAPM. The ques- tionnaire leaves open issues of poor self-report and awareness. In addition, there were relatively few indi- viduals with TBI included, and no imaging data were available to know the specific areas of damage to the brain, thus any suggestions about different regions of prefrontal cortex having different functions are purely speculative. Moreover, the TBI sample likely contains individuals with a range of different cognitive profiles and brain regions affected. Similarly, the individuals with TBI were given only limited neuropsychological measures, thus we are not able to characterize the nature of their cognitive deficits or the ways that the PM deficit fits into an overall pattern. Future studies with larger samples could take advantage of regression analyses to try to tease out whether there are separate and unique aspects of PM being measured by these tasks.

These results are more consistent with the multiprocess theory of PM than the PAM theory, given that the performance of the HA group on the 2-min cues of the MIST are almost perfect, despite adequate attention paid to the ongoing task, and the effect of related pairs being superior to unrelated on the laboratory measure. They support previous findings of PM deficits in TBI, with time-based items being more impaired than event-based items.

In sum, the results of this study suggest that different aspects of PM performance may be being measured by different assessment techniques. Thus, further researchis needed to determine whether there are unique factors that might be separable in PM processing that have not yet been examined, as our data seem to go beyond the usual distinctions such as time-based versus event-based. Research is needed to determine which specific factors are being measured by each unique PM task used. Clinical assessments should take this into account when interpreting the effects of PM deficits on daily life and be aware that a single PMclinicalmeasure may onlybetargeting a small subset of PM performance. Moreoever, self-report measures, in particular, seem to have a low statistical relationship with objective measures and should be interpreted within that context. Importantly, different clinical populations who have PM impairments may be using different cognitive processes than those who have intact PM performance and thus may show unique and specific loadings on individual factors. Further study of the differences between these techniques could yield important theoretical information about the nature of PM as well.

\section{Acknowledgments}

The authors would like to thank Gayna Swart for help with statistical analyses.

\section{Disclosure statement}

No potential conflict of interest was reported by the authors.

\section{References}

Banich, M., Milham, M., Atchley, R., Cohen, N., Webb, A., Wszalek, T., Magin, R. (2000). fMRI studies of stroop tasks reveal unique roles of anterior and posterior brain systems in attentional selection. Journal of Cognitive Neuroscience, 12, 988-1000.

Beck, A.,\& Steer, R. (1990). Manual for the beck anxiety depres- sion inventory. San Antonio, TX: Psychological Corporation.

Beck, A. T., \& Steer, R. A. (1987). Manualfor the revised beck depression inventory. San Antonio, TX: Psychological Corporation.

Burgess, P., Scott, S., \& Frith, C. (2003). The role of the rostral frontal cortex (area 10) in prospective memory: A lateral versus medial dissociation. Neuropsychologia, 41, 906-918. doi:10.1016/S0028-3932(02)00327-5

Carey, C. L., Woods, S. P., Rippeth, J. D., Gonzalez, R., Moore, D. J., Marcotte, T. D., et al. (2004). Initial validation of a screening battery for the detection of HIV-associated cognitive impairment. The Clinical Neuropsychologist, 18, 234248. doi:10.1080/13854040490501448

Chau, L. T., Lee, J. B., Fleming, J., Roche, N., \& Shum, D. (2007). Reliability and normative data for the Comprehensive Assessment of Prospective Memory (CAPM). Neuropsychological Rehabilitation, 17(6), 707722. doi:10.1080/09602010600923926

Chi, S., Rabin, L., Aronov, A., Fogel, J., Kapoor, A., \& Wang, C. (2014). Differential focal and nonfocal propsective memory accuracy in a demographically diverse group of nondemented community-dwelling older adults. Journal of the International Neuropsychological Society, 20, 
1015- 1027. doi:10.1017/S1355617714000964

Crawford, J. R., Smith, G., Maylor, E. A., Della Sala, S., \& Logie, R. H. (2003). The Prospective and Retrospective Memory Questionnaire (PRMQ): Normative data and latent structure in a large non-clinical sample. Memory, 11(3), 261-275. doi:10.1080/09658210244000027

d'Ydewalle, G., Bouckaert, D., \& Brunfaut, E. (2001). Age- related differences and complexity of ongoing activities in time- and event-based prospective memory. American Journal of Psychology, 114, 411-423. doi: $10.2307 / 1423688$

Delprado, J., Kinsella, G., Ong, B., Pike, K., Ames, D., Storey, E., . . Rand, E. (2012). Clinical measures of prospective memory in amnestic mild cognitive impairment. Journal of the International Neuropsychological Society, 18(2), 295-304. doi:10.1017/S135561771100172X

Dobbs, A. R., \& Reeves, M. B. (1996). Prospective memory: More than memory. In M. A. Brandimonte, G. O. Einstein, \&M. A. McDaniel (Eds.), Prospective memory: Theory and applications (pp. 199-225). Mahwah, NJ: Erlbaum.

Einstein, G. O., \& McDaniel, M. A. (1990). Normal aging and prospective memory. Journal of Experimental Psychology: Learning, Memory, and Cognition, 16, 717-726.

Einstein, G. O., \& McDaniel, M. A. (2005). Prospective memory: Multiple retrieval processes. Current Directions in Psychological Science, 14(6), 286-290. doi:10.1111/ j.0963-7214.2005.00382.x

Einstein, G. O., McDaniel, M. A., Richardson, S. L., Guynn,

M. J., \& Cunfer, A. R. (1995). Aging and prospective memory: Examining the influences of self-initiated retrieval processes. Journal of Experimental Psychology: Learning, Memory, and Cognition, 2, 996-1007.

Ellis, J., \& Freeman, J. (2008). Ten years on: Realizing delayed intentions. In M. Kliegel, M. McDaniel, \& G. Einstein (Eds.), Prospective memory: Cognitive, neuroscience, developmental and applied perspectives (pp. 1-28). New York: Lawrence Erlbaum Associates.

Ellis, J. A. (1996). Prospective memory or the realisation of delayed intentions: A conceptual framework for research. In M. Brandimonte, G. O. Einstein, \& M. A. McDaniel (Eds.), Prospective memory: Theory and applications (pp. 1-22). Hillsdale,NJ: Erlbaum.

Fleming, J., Doig, E., \& Katz, N. (2000). Beyond dressing and driving: Using occupation to facilitate community integra- tion in neurorehabilitation. Brain Impairment, 1, 141-150. doi:10.1375/brim.1.2.141

Fleming, J., Kennedy, S., Fisher, R., Gill, H., Gullo, M., \& Shum, D. (2009). Validity of the Comprehensive Assessment of Prospective Memory (CAPM) for use with adults with traumatic brain injury. Brain Impairment, 10 (1), 34-44. doi:10.1375/brim.10.1.34

Fleming, J. M., Strong, J., \& Ashton, R. (1996). Self-aware- ness of deficits in adults with traumatic brain injury: How best to measure? Brain Injury, 10(1), 1-16.

Foster, E., McDaniel, M., Repovs, G., \& Hershey, T. (2009).

Prospective memory in Parkinson's disease across laboratory and self-reported everyday performance. Neuropsychology, 23,347-358. doi:10.1037/a0014692

Hannon, R., Adams, P., Harrington, S., Fries-Dias, C., \& Gipson, M. T. (1995). Effects of brain injury and age on self-rating and performance. Rehabilitation Psychology, 40 (4), 289-298. doi:10.1037/0090-5550.40.4.289

Harris, B., Jette, A., Campion, E., \& Cleary, P. (1986). Validity of self-report measures of functional disability. Topics in Geriatric Rehabilitation, 1, 31-41. doi:10.1097/ 00013614-198604000-00005

Huang, J., Fleming, J., Pomery, N., O’Gorman, J., Chan, R., \& Shum, D. (2014). Perceived importance of prospective memory failures in adults with traumatic brain injury. Neuropsychological Rehabilitation, 24, 61-70. doi:10.1080/ 09602011.2013 .854723

Kiernan, R. J., Mueller, J., Langston, J. W., \& Van Dyke, C. (1987). The neurobehavioral cognitive status examination, A brief but differentiated approach to cognitive assess- ment. Annals of Internal Medicine., 107, 481-485. doi:10.7326/0003-4819-107-4-481

Kvavilashvili, L., Cockburn, J., \& Kornbrot, D. (2012). Prospective memory and ageing paradox with event-based tasks: A study of young, young-old, and old-old participants. The Quarterly Journal of Experimental Psychology, 66, 864- 875. doi:10.1080/17470218.2012.721379

Leung, H., Skudlarski, P., Gatenby, J., Peterson, B., \& Gore, J. (2000). An event-related functioning MRI study of the stroop color word interference task. Cerebral Cortex, 10, 552-560. doi:10.1093/cercor/10.6.552

Man, D., Fleming, J., Hohaus, L., \& Shum, D. (2011). Development of the brief assessment of prospective memory for use with traumatic brain injury populations. Neuropsychological Rehabilitation, $21,884-898$. doi:10.1080/09602011.2011.627270

Mathias, J. L., \& Mansfield, K. M. (2005). Prospective and declarative memory problems following moderate and severe traumatic brain injury. Brain Injury, 19(4), 271-282. doi:10.1080/02699050400005028

Maylor, E. A., Smith, G., Della Sala, S., \& Logie, R. H. (2002). Prospective and retrospective memory in normal aging and dementia: An experimental study. Memory \& Cognition, 30, 871-884. doi:10.3758/BF03195773

McDaniel, M., \& Einstein, G. (2011). The neuropsychology of prospective memory in normal aging: A componential approach. Neuropsychologia,49,2147-2155. doi:10.1016/j. neuropsychologia.2010.12.029

McDaniel, M., Guynn, M., Einstein, G., \& Breneiser, J. (2004). Cue-focused and reflexive-associative processes in 
prospective memory retrieval. Journal of Experimental Learning Memory and Cognition, 30, 605-614. doi:10.1037/0278-7393.30.3.605

Mioni, G., Rendell, P., Henry, J., Cantagallo, A., \& Stablum, F. (2013). An investigation of prospective memory functions in people with traumatic brain injury using Virtual Week. Journal of Clinical and Experimental Neuropsychology,35, 617-630. doi:10.1080/13803395.2013.804036

Mioni, G., Stablum, F., McClintock, S., \& Cantagallo, A. (2012). Time-based prospective memory in severe trau- matic brain injury patients: The involvement of executive functions and time perception. Journal of the International Neuropsychological Society, 18, 697-705. doi:10.1017/ S1355617712000306

Mitchell, M., Miller, S., Woodard, J., Davey, A., Martin, P., Burgess, M., \& Poon, L. (2011). Regression-based estimates of observed functional status in centenarians. Gerontologist, 51, 179-189. doi:10.1093/geront/gnq087

Molander, B., \& Arar, L. (1998). Norms for 439 action events: Familiarity, emotionality, motor activity, and memorability. Scandanavian Journal of Psychology, 39(4), 275-300. doi:10.1111/1467-9450.00087

Nelson, D. L., McEvoy, C. L., \& Schreiber, T. A. (1998). The University of South Florida word association, rhyme, and word fragment norms. http://www.usf.edu/FreeAssociation/.

Neulinger, K., Oram, J., Tinson, H., O’Gorman, J., \& Shum, D. (2015, July). Prospective memory and frontal lobe function. Aging, Neuropsychology, and Cognition, 35,(Eptlb Ahead of Print).

Overall, J. E., \& Gorham, D. R. (1962). The brief psychiatric rating scale. Psychological Reports, 10, 799-812. doi:10.2466/pr0.1962.10.3.799

Pavawalla, S., Schmitter-Edgecombe, M., \& Smith, R. (2012). Prospective memory after moderate-to-severe traumatic brain injury: A multinomial modeling approach. Neuropsychology, 26, 91-101. doi:10.1037/a0025866

Paxton, J., \& Chiaravalloti, N. (2014). Rule monitoring ability predicts event-based prospective memory performance in individuals with TBI. Journal of the International Neuropsychological Society, 20, 673-683. doi:10.1017/ S1355617714000575

Pereira, A., Ellis, J., \& Freeman, J. (2012). Is prospective memory enhanced by cue-action semantic relatedness and enactment at encoding? Consciousness and Cognition, 21, 1257-1266. doi:10.1016/j.concog.2012.04.012

Radford, K. A., Lah, S., Say, M. J., \& Miller, L. A. (2011). Validation of a new measure of prospective memory: The royal prince alfred prospective memory test. The Clinical Neuropsychologist, 25(1), 127-140. doi: $10.1080 /$ 13854046.2010 .529463

Raskin, S., Buckheit, C., \& Sherrod, C. (2010). Memory for Intentions Test: Manual. Lutz, FL: Psychological Assessment Resources.

Raskin, S., Buckheit, C., \& Waxman, A. (2012). Effect of type of cue, type of response, time delay and two different ongoing tasks on prospective memory functioning after acquired brain injury. Neuropsychological Rehabilitation, 22(1), 40-64. doi:10.1080/09602011.2011.632908

Raskin, S., Maye, J., Rogers, A., Correll, D., Zamroziewicz, M., \& Kurtz, M. (2014). Prospective memory in schizophrenia: Relationship to medication management skills, neurocogni- tion, and symptoms in individuals with schizophrenia. Neuropsychology, 28, 359-365. doi:10.1037/neu0000040

Raskin, S., Smith, M., Mills, G., Pedro, C., \& Zamroziewicz, M. (2017). Prospective memory intervention using visual imagery in individuals with brain injury. Neuropsychological Rehabilitation. Advanced online pub- lication. doi:10.1080/09602011.2017.1294082

Raskin, S. A. (2009). Memory for intentions screening test: Psychometric properties and clinical evidence. Brain Impairment, 10(1), 23-33. doi:10.1375/brim.10.1.23

Raskin, S. A., \& Sohlberg, M. M. (2009). Prospective memory intervention: A review and evaluation of a pilot restorative intervention. Brain Impairment, 10(1), 76-86. doi:10.1375/ brim.10.1.76

Raskin, S. A., Woods, S. P., Poquette, A. J., McTaggart, A. B., Sethna, J., Williams, R. C., \& Tröster, A. I. (2011). A differential deficit in time- versus event-based prospective memory in Parkinson's disease. Neuropsychology, 25(2), 201-209. doi:10.1037/a0020999

Rendell, P. G., \& Henry, J. (2009). A review of virtual week for prospective memory assessment: Clinical implications. Brain Impairment, 10, 14-22. doi:10.1375/brim.10.1.14

Rendell, P. G., \& Thomson, D. M. (1999). Aging and pro- spective memory: Differences between naturalistic and laboratory tasks. The Journals of Gerontology: Series B: Psychological Sciences and Social Sciences, 54B(4), 256- 269. doi:10.1093/geronb/54B.4.P256

Roche, N., Fleming, J., \& Shum, D. (2002). Self-awareness of prospective memory failure in adults with traumatic brain injury. Brain Injury, 16, 931-945. doi:10.1080/ 02699050210138581

Roche, N., Moody, A., Szabo, K., Fleming, J., \& Shum, D. (2007). Prospective memory in adults with traumatic brain injury: An analysis of perceived reasons for remembering and forgetting. Neuropsychological Rehabilitation, $17,314-$ 
334. doi:10.1080/09602010600831004

Scullin, M. K., McDaniel, M. A., \& Shelton, J. T. (2013). The dynamic multiprocess framework: Evidence from prospective memory with contextual variability. Cognitive Psychology, $67 \quad(1), \quad 55-71$. doi:10.1016/j.cogpsych.2013.07.001

Sherer, M., Boake, C., Levin, E., \& Silver, B. (1998).

Characteristics of impaired awareness after traumatic brain injury. Journal of the International Neuropsychological Society, 4, 380-387.

Shum, D., Levin, H., \& Chan, R. (2011). Prospective memory in patients with closed head injury: A review. Neuropsychologia, 49, 2156-2165. doi:10.1016/j. neuropsychologia.2011.02.006

Shum, D., Ungvari, G., Tang, W., \& Leung, J. (2004). Performance of schizophrenia patients on time--, event-, and activity-based prospective memory tasks. Schizophrenia Bulletin, 30, 693-701. doi:10.1093/oxfordjournals.schbul.a007123

Shum, D., Valentine, M., \& Cutmore, T. (1999). Performance of individuals with severe long-term traumatic brain injury on time-, event-, and activity-based prospective memory tasks. Journal of Clinical and Experimental Neuropsychology, 21(1), 49-58. doi:10.1076/ jcen.21.1.49.943

Smith, R. (2003). The cost of remembering to remember in event-based prospective memory: Investigating the capacity demands of delayed intention performance. Journal of Experimental Psychology: Learning, Memory and Cognition, 29, 347-361.

Teasdale, G., \& Jennett, B. (1974). Assessment of coma and impaired consciousness. A Practical Scale, 2(7872), 81-84. Twamley, E. W., Woods, S. P., Zurhellen, C. H., Vertinski, M., Narvaez, J. M., Mausbach, B. T., ... Jeste,

D. (2008).

Neuropsychological substrates and everyday functioning implications of prospective memory impairment in schizophrenia. Schizophrenia Research, 106, 42-49. doi:10.1016/j.schres.2007.10.030

Uttl, B., \& Kibreab, M. (2011). Self-report measures of pro- spective memory are reliable but not valid. Canadian Journal of Experimental Psychology/Revue canadienne de psychologie expérimentale, 65(1), 57-68. doi:10.1037/ a0022843

Ward, H., Shum, D., McKinlay, L., Baker, S., \& Wallace, G. (2007). Prospective memory and pediatric traumatic brain injury: Effects of cognitive demand. Childhood Neuropsychology, 13, 219-239. doi:10.1080/ 09297040600910003

Waugh, N. (1999). Self-report of the young, middle-aged, young- old and old-old individuals on prospective memory function- ing. Unpublished honours thesis, School of Applied Psychology, Griffith University, Brisbane, Australia.

Wilson, B. A., Emslie, H., Foley, J., Shiel, A., Watson, P., Hawkins, K., . . . Evans, J. J. (2005). The Cambridge Prospective Memory Test (CAMPROMPT). London: Harcourt Assessment.

Woods, S. P., Dawson, M. S., Weber, E., Gibson, S., Grant, I., \& Atkinson, J. H. (2009). Timing is everything: Antiretroviral nonadherence is associated with impair- ment in time-based prospective memory. Journal of the International Neuropsychological Society, 15, 42-52. doi:10.1017/S1355617708090012

Woods, S. P., Moran, L., Dawson, M., Carey, C., Grant, I., \& Group, H. N. R. C. (2008). Psychometric characteristics of the memory for intentions screening test. Clinical Neuropsychology, $22, \quad 864-878$. doi:10.1080/ 13854040701595999

Woods, S. P., Weber, E., Cameron, M., Dawson, M., \& Grant, I. (2010). HIV infection is associated with comparable deficits in time- and event-based prospective memory. Archives of Clinical Neuropsychology, 25, 543-543.

Woods, S. P., Weinbom, M., Velnoweth, A., Rooney, A., \& Bucks, R. (2012). Memory for intentions is uniquely associated with instrumental activities of daily living in older adults. Journal of the International Neuropsychological Society, 18, 134-138. doi:10.1017/ S1355617711001263 